\title{
Horizons/Théâtre
}

Revue d'études théâtrales

10-11 | 2017

Genre et arts vivants

\section{Quand les femmes entrent en piste ! Domptage et émancipation féminine au passage $\mathrm{du}_{\mathrm{XIX}} \mathrm{e}^{\mathrm{e}}$ au $\mathrm{xx}^{\mathrm{e}}$ siècle}

\section{Charlotte Foucher Zarmanian}

\section{(2) OpenEdition}

\section{Journals}

Édition électronique

URL : https://journals.openedition.org/ht/571

DOI : $10.4000 /$ ht.571

ISSN : 2678-5420

\section{Éditeur}

Presses universitaires de Bordeaux

\section{Édition imprimée}

Date de publication : 1 juillet 2017

Pagination : 240-258

ISSN : 2261-4591

Référence électronique

Charlotte Foucher Zarmanian, « Quand les femmes entrent en piste ! Domptage et émancipation féminine au passage du xix ${ }^{\mathrm{e}}$ au xx ${ }^{\mathrm{e}}$ siècle », Horizons/Théâtre [En ligne], 10-11 | 2017, mis en ligne le 01 juillet 2018, consulté le 15 février 2023. URL : http://journals.openedition.org/ht/571 ; DOI : https:// doi.org/10.4000/ht.571

\section{c) (†) $\ominus$}

Creative Commons - Attribution - Pas d'Utilisation Commerciale - Pas de Modification 4.0 International - CC BY-NC-ND 4.0

https://creativecommons.org/licenses/by-nc-nd/4.0/ 


\section{CHARLOTTE FOUCHER ZARMANIAN}

Docteure en Histoire de l'art, Charlotte Foucher Zarmanian est chargée de recherches au CNRS, affiliée au Laboratoire d'Études de Genre et de Sexualité (LEGS - UMR 8238). Elle a soutenu une thèse de doctorat sur les femmes artistes dans les milieux symbolistes en France, qui a été récompensée du Prix du musée d'Orsay 2013 permettant la publication d'un essai chez Mare \& Martin en 2015. Ses recherches portent actuellement sur les savoirs artistiques des femmes ( $\mathrm{xx}$ - $\mathrm{xVIII}$ e siècles), ainsi que sur les interactions pouvant exister entre les femmes et les musées. Elle coordonne à ce sujet, avec Hélène Marquié, l'axe "Genre, création artistique, matrimoine » dans le cadre du projet lauréat de l'appel à projets lancé par l'Université Paris Lumières et intitulé « Genre et transmission : pour une autre archéologie du genre » (2016-2019).

Mail : charlotte.foucher@legs.cnrs.fr

Résumé: Au XIXe siècle, la majeure partie des représentations de saltimbanques repose sur une stricte répartition des genres, où l'homme pratique le domptage quand la femme est cantonnée aux rôles passifs et érotisés de l'acrobate ou de la voltigeuse. Cette partition bien orchestrée semble, cependant, progressivement se troubler, et ce, notamment avec l'arrivée de la dompteuse, qui envahit les pistes de cirque, la littérature et la culture visuelle de la fin du xIXe siècle. L'objectif de cet article est $d$ 'interroger la spécificité de cette figure liée aux arts vivants, en prenant le soin de mettre en lumière les nombreux paradoxes qu'elle soulève. Car, si la dompteuse peut illustrer symboliquement la nouvelle émancipation conquise par les femmes sur les territoires artistiques et intellectuels, elle semble en

Abstract: In the nineteenth century, most of the representations of circus entertainers were based on a strict gendered division: men practiced taming whereas women were confined to the passive and erotic roles of acrobat or aerobatics. However, this well-orchestrated partition seems to be disturbed, notably by the arrival of the woman tamer, which invades the circus tracks, literature and visual culture of the late nineteenth century. This article aims at examining the specificity of this figure linked to the performing arts and to highlight the paradoxes it raises. If the woman tamer can symbolically illustrate the new emancipation conquered by women in the artistic and intellectual territories, it seems at the same time to lead a number of stereotypes même temps reconduire un certain nombre de stéréotypes alimentant la psychopathologie féminine. Partant de cette ambivalence entre le masculin et le féminin, entre la convenance et la transgression, l'analyse de multiples portraits de dompteuses autour de 1900 - fictifs et réels - permettra de montrer pourquoi et comment certaines femmes, étrangères aux milieux circassiens, s'emparent de cette figure pour repousser les limites d'une norme féminine, façonner d'autres catégories identitaires et confirmer une crise de la masculinité déjà en marche.

Mots-clés : dompteuse, émancipation des femmes, genre, représentations, culture visuelle

reinforcing female psychopathology. Looking at his ambivalence between masculinity and femininity, suitability and transgression, the analysis of the multiple portraits of tamers around 1900 - fictional and real - will show why and how several women, foreign to circassian milieux, seize this figure to push off the limits of a feminine norm, to shape other identity categories and to confirm a masculinity crisis in progress.

KeYWORDS: woman tamer, feminine emancipation, gender, representations, visual culture 


\section{Quand les femmes entrent en piste ! Domptage et émancipation féminine au passage du XIX au $\mathrm{XX}^{\mathrm{e}}$ siècle $^{1}$}

Jusqu'au milieu du XIX ${ }^{\mathrm{e}}$ siècle, la majeure partie des descriptions et représentations de saltimbanques repose sur une stricte répartition des genres où l'homme pratique le domptage quand la femme est cantonnée aux rôles passifs et érotisés de l'acrobate, de l'écuyère, de la funambule ou de la voltigeuse $^{2}$. Le cliché masculin de l'autorité fait globalement face à celui, considéré comme plus féminin, de la souplesse ou de l'agilités. Pourtant, cette partition des rôles bien orchestrée se trouble progressivement, notamment du fait de l'apparition des dompteuses dans les années 1840, dix ans après les premiers dompteurs. Gagnant significativement la culture populaire, visuelle et textuelle de la fin du XIX ${ }^{e}$ siècle (peinture, affiche, photographie, presse satirique, presse féministe, roman, théâtre), la dompteuse - originellement associée aux arts du cirque - subvertit les archétypes ordinaires de domination et devient aussi, plus discrètement, une figure paradigmatique pour de nombreuses femmes soucieuses de revendiquer une plus grande indépendance et souveraineté.

L'objectif de cet article est d'interroger la spécificité de cette figure liée aux arts vivants, en prenant le soin de mettre en lumière les nombreux paradoxes qu'elle soulève. Car, si la dompteuse peut illustrer symboliquement la nouvelle émancipation conquise par les femmes sur les territoires artistiques et intellectuels, elle semble en même temps reconduire un certain nombre de stéréotypes alimentant les psychopathologies féminines. Partant de cette ambivalence entre le masculin et le féminin, entre la convenance et la transgression, nous analyserons de multiples portraits de dompteuses autour de 1900 - fictifs et réels - afin de montrer pourquoi et comment certaines femmes, étrangères aux milieux circassiens, s'emparent de cette figure pour repousser les limites d'une norme féminine, façonner d'autres catégories identitaires et confirmer une crise de la masculinité déjà en marche. 


\section{La dompteuse, une figure ambivalente entre féminin et masculin}

L'ambivalence de la dompteuse, adoptant à la fois les stéréotypes du féminin et du masculin, permet de poser trois types de questions : comment parvient-elle à cette activité ? Comment l'exerce-t-elle ? Et quel portrait physique fait-on d'elle?

\section{Les origines : femme sauvage ou héritière patriarcale ?}

Dans son ouvrage paru en 1928, Les Dompteurs ou La Ménagerie des origines à nos jours, Henry Thétard - lui-même dompteur de fauves et personnalité reconnue de l'histoire du cirque - précise que la première femme à pratiquer le domptage serait madame Leprince, une Française, la première à entrer dans une cage aux fauves en $1839^{4}$. Thétard explique notamment que madame Leprince possède un don particulier : celui de comprendre les animaux et de déceler leurs tempéraments. Certes, plusieurs hommes disposent aussi de cette capacité dite « instinctive », mais ne peut-on pas faire l'hypothèse qu'elle se teinte d'une dimension autre lorsqu'elle se trouve détenue par une femme? Pensons ainsi aux nombreux auteurs de traités scientifiques qui conviennent ensemble d'une proximité anthropologique, psychologique et même physique de la femme avec l'animal. Convaincus de l'infériorité des femmes, les médecins italiens Cesare Lombroso et Guglielmo Ferrero s'intéressent, par exemple, dans leur célèbre ouvrage La Donna deliquente, la prostitute e la donna normale, publié en Italie en 1893, puis rapidement traduit et diffusé dans toute l'Europe, aux crimes commis respectivement par les femelles dans le règne animal et à ceux des êtres dits sauvages et des femmes dites primitives. Ils rapprochent les comportements des femmes criminelles de ceux de la vie sauvage $^{5}$. La mise en évidence d'une bestialité typiquement féminine fournit un argument de choix aux deux médecins : la femme serait un être intrinsèquement criminel et sexuel, sur lequel la maternité jouerait un rôle calmant et salvateur ${ }^{6}$. La bestialisation de la femme s'illustre aussi dans d'autres traités, physiognomoniques cette fois, comme dans celui du craniologue allemand Carl Vogt qui précise que « lorsqu'est suggéré un rapprochement avec le genre animal, la femme s'en rapproche davantage que l'homme $[\ldots]^{7} \gg$. La puissance tautologique de ces textes scientifiques, faisant à l'époque autorité, est telle que les représentations artistiques s'en emparent pour pointer visuellement l'analogie entre la femme criminelle et sexuelle, et le fauve. La représentation de l'héroïne Salomé est en ce sens tout à fait significative. Elle adopte un sourire de carnassière et une attitude féline dans le tableau d'Henri Régnault, qui revendique lui-même dans une lettre à Cazalis cette proximité avec la bête sauvage : «La férocité caressante est le fond de sa nature, 
et c'est bien une sorte de panthère noire, apprivoisée mais toujours sauvage cruelle $^{8}$. » Elle est encore fièrement accompagnée d'une panthère noire dans l'œuvre de Pierre Amédée Marcel-Beronneau, élève de Gustave Moreau, qui représente la femme fatale en triomphe devant Saint Jean-Baptiste décapité. Outre ces implications dans la culture visuelle de l'époque, l'argument naturaliste se retrouve plus concrètement dans le cas de la dompteuse Nouma Hawa qui dans ses mémoires réactive quelques-unes de ces considérations sur la femme sauvage, et prolonge l'exotisme déjà entrevu chez Salomé. La dompteuse raconte en effet son adolescence passée au pays des Ounemis en Afrique centrale où, en tant que seule blanche, elle apprend en compagnie de ces « sauvages », « nègres » ou « barbares », comme elle les appelle, l'art de charmer les serpents et surtout de dompter les fauves ${ }^{9}$.

À l'opposé de Nouma Hawa, qui se forme à l'art du domptage en regardant et en reproduisant les gestes des Ounemis, d'autres dompteuses sont héritières de la profession paternelle. C'est le cas de Gladys, héroïne d'une comédie dramatique intitulée Gladys la dompteuse, qui apprend le métier auprès de son père directeur d'un cirque ${ }^{10}$, et dont l'histoire a peut-être été inspirée du cas réel d'Olga Jeannet qui arrive également au domptage par son père, Auguste Jeannet, propriétaire de la ménagerie franco-suisse. Il la fait entrer dans la cage aux fauves alors qu'elle n'est qu'une petite fille. Cet « événement » aurait convaincu Olga Jeannet de sa vocation : elle sera dompteuse, et prendra la suite de son père et de son oncle Fritz Jeannet, également dresseur d'ours, eux-mêmes descendant d'une longue lignée de dresseurs et de dompteurs.

\section{La pratique du domptage : transmission ou domination ?}

Comme l'entrée dans le métier, l'exercice même du domptage se teinte d'une ambivalence entre le masculin et le féminin. Dans la pratique de son art, et de manière à ne pas perturber les convenances, la dompteuse semble en effet, d'abord, réactiver les qualités de transmission et d'éducation traditionnellement associées au féminin. L'historien Paul Rousselot, auteur d'une Histoire de l'éducation des femmes en France parue en 1883, explique en effet que « [1] a figure de la mère, institutrice née domine toute la pédagogie féminine du XIX ${ }^{\mathrm{e}}$ siècle $^{11} \gg$. Ce type de discours essentialisant semble être relayé par cette image représentant la dompteuse Claire Heliot, bien connue pour son empathie à l'égard des animaux, et occupée ici à nourrir ses lions ${ }^{12}$. 


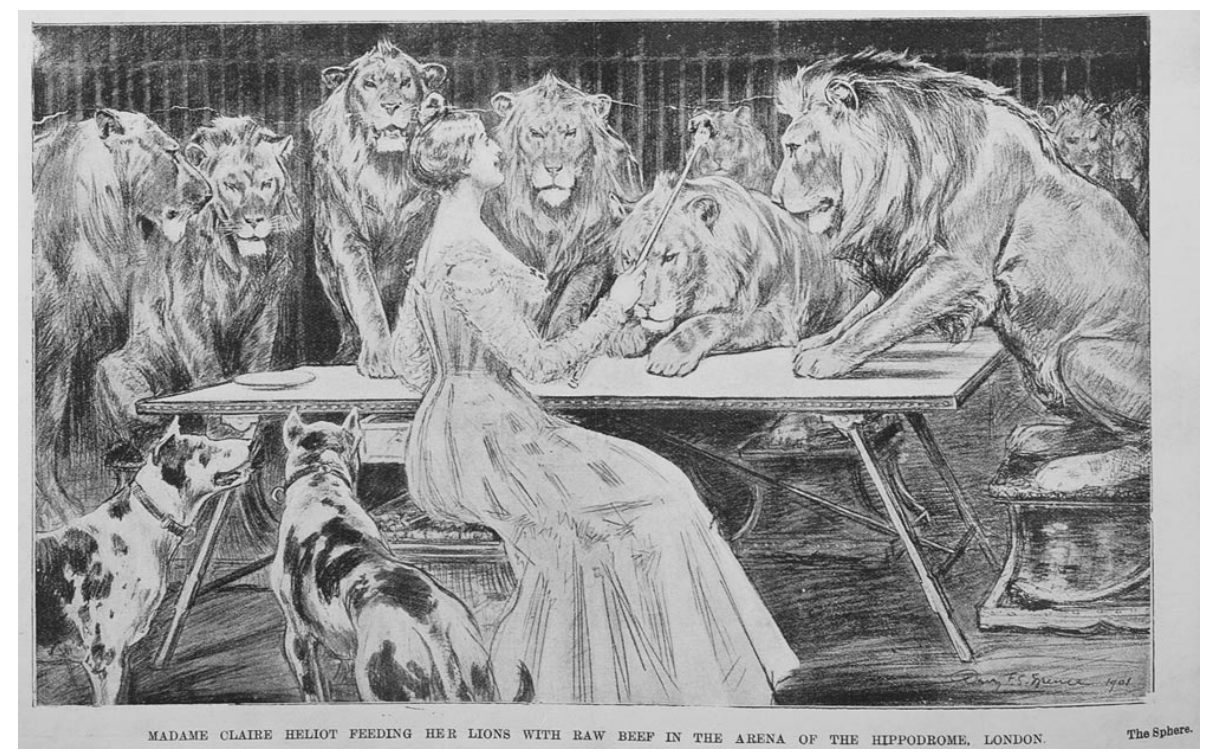

Madame Claire Héliot donnant à manger à ses lions, Londres, [s. d.].

La table et les tabourets sur lesquels ils reposent debout contribuent à humaniser ces bêtes qui, loin de la sauvagerie indisciplinée pouvant leur être associée, ressemblent à des élèves dociles et attentifs aux recommandations de leur maîtresse. Confrontée majoritairement à des animaux impressionnants, capricieux, difficiles à amadouer ${ }^{13}$, la dompteuse tantôt pratique le domptage avec douceur ${ }^{14}$, tantôt combat l'animal sauvage dans un corps-à-corps plus agressif.

Car si la dompteuse s'emploie à tisser un lien presque maternel avec les animaux, elle peut aussi incarner de manière plus subversive une figure de domination, illustrant littéralement la définition de « dompter » extraite du dictionnaire Littré (qui dans ses entrées ignore, par ailleurs, la dompteuse au profit du dompteur), à savoir faire fléchir, assujettir, faire perdre son caractère indépendant et sauvage ${ }^{15}$. C'est le cas de Norah la dompteuse qui dans une pièce de théâtre éponyme met en déroute la virilité du personnage mascu$\operatorname{lin}^{16}$. Dans le deuxième acte, Norah explique à deux journalistes qui viennent s'entretenir avec elle qu'elle n'a jamais eu d'amants, car elle impose au prétendant d'entrer avec elle dans la cage aux fauves pour prouver son amour ${ }^{17}$. Passionnément épris de Norah, le magistrat Moulineau ose relever le défi et s'exécute avec difficulté - mais en vain - à cette demande : 
MOULINEAU - Voilà... Oh ! les sales bêtes, mon Dieu, les sales bêtes !... Vous veniez de me quitter..., aïe!

NORAH - Vous souffrez

MOULINEAU - Comme une brute !... vous veniez de me quitter et je me promenais dans le jardin, rêvant à vous, Norah, à vos charmes, à vos grâces, à tous ces trésors..., aïe. [...] Tout à coup, je pris une résolution que je ne crains pas de qualifier de viril.

NORAH - Vous êtes entré dans la cage?

MOULINEAU - Pas tout de suite... J'ai d'abord essayé de me familiariser avec vos... Oh! les sales bêtes! mon Dieu, les sales bêtes! Leur parlant amicalement, doucement, leur parlant enfin, comme un père parlerait à ses enfants... Puis pour les séduire tout à fait, je leur ai distribué quelques morceaux de sucre que j'avais par hasard sur moi.

NORAH - Ils n'aiment que la viande...

MOULINEAU - Je m'en suis aperçu... Cependant, ils avaient paru touchés de mon attention, sensibles à la politesse que je leur faisais... Aussi, profitant de leurs bonnes dispositions..., apparentes..., hélas ! et surtout songeant à la récompense promise, je n'hésitai plus... J'ouvris toute grande la porte de la cage et... oh! les sales bêtes, mon Dieu ! les sales bêtes !... La porte était à peine ouverte qu'elles se précipitaient toutes sur moi, furieuses et rugissantes...

NORAH - Il fallait les braver, les dominer du regard.

MOULINEAU - Impossible !... J'étais entré de dos, à reculons, justement parce que leurs regards m'intimidaient. J'ai essayé de me retourner après... mais trop tard... Ah! dame, c'est la première fois que je fais ça et quand on n'en a pas l'habitude... Je ne l'aurai jamais..., vous savez..., jamais... Et toute ma vie je me rappellerai ce moment-là $\dot{1}^{18}$.

Cet extrait du vaudeville repose sur un rapport oppositionnel entre l'amant, peureux, faible, et la dompteuse triomphant par son courage et sa force. Comme une façon aussi, plus largement, d'intensifier le spectaculaire caractérisant ces lieux du cirque, la femme dont on vante plus volontiers la faiblesse se dote chez la dompteuse d'une force héroïque, impressionnante, qui semble excéder la « nature » féminine. Les accidents ne sont d'ailleurs pas rares à l'époque et font souvent l'objet de unes à sensation dans la presse contemporaine, comme celle montrant la Goulue à la suite d'une grave morsure en 1904 ou encore celle représentant Rosita Gondolfo morte quelques années plus tôt à Grenoble égorgée par une lionne ${ }^{19}$. 


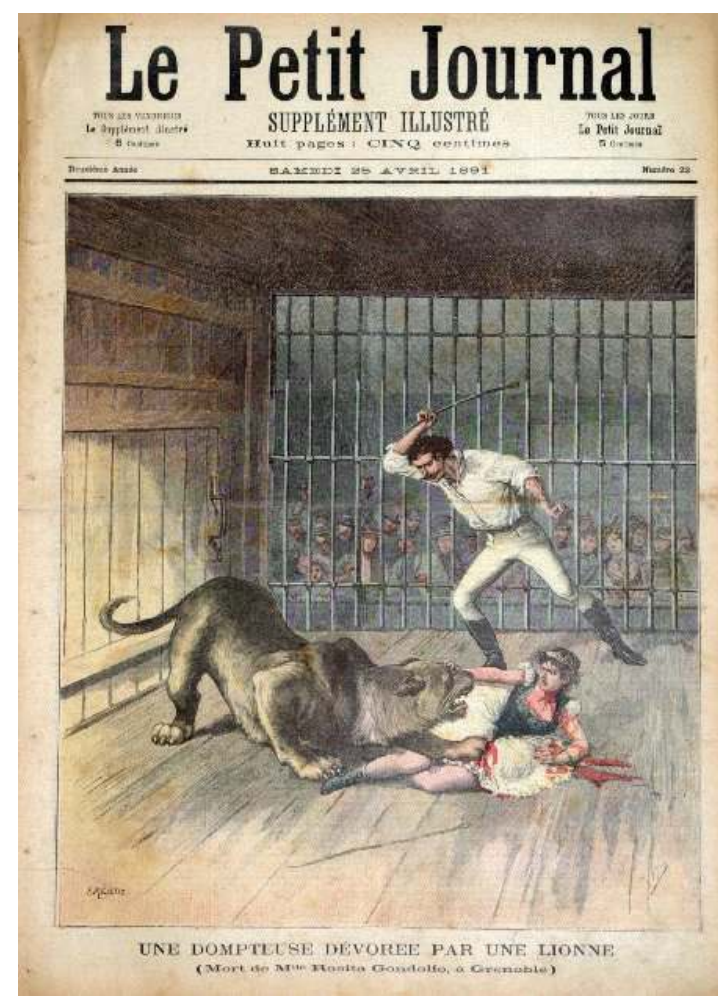

« Une dompteuse dévorée par une lionne (Mort de Mlle Rosita Gondolfo à Grenoble) », Le Petit Journal. Supplément illustré, samedi 25 avril 1891, une.

Sous des apparences souvent racoleuses jouant des ressorts dramatiques, ces illustrations soutiennent le courage de ces femmes qui sont en permanence confrontées au danger et risquent très souvent leur vie dans l'exercice de leur profession.

\section{Les caractéristiques physiques de la dompteuse : repenser la féminité}

Dans la majorité des représentations de dompteuses, le portrait physique déroge rarement aux stéréotypes attendus de la féminité. Ces femmes sont belles et séduisent bien souvent les hommes ${ }^{20}$, à l'instar de Cornaline hérö̈ne du roman de Fortuné de Boisgobey :

Il y avait même, l'année dernière, une ménagerie fort bien montée en bêtes fauves qui travaillaient sous la cravache d'une femme, et par extraordinaire cette femme était très belle. [... C C'était une grande fille bien découplée, brune de peau, avec des yeux splendides, des yeux d'Orientale, et une profusion de cheveux noirs qui retombaient sur ses épaules et que, pendant ses exercices, elle secouait comme 
une crinière. Son maillot moulait des formes robustes, mais élégantes. Ses pieds, chaussés de brodequins, étaient petits et cambrés ; et qualité plus rare chez les foraines, elle soignait ses mains ${ }^{21}$.

Une affiche montrant la dompteuse Claire Heliot se conforme aussi à une traditionnelle norme féminine.

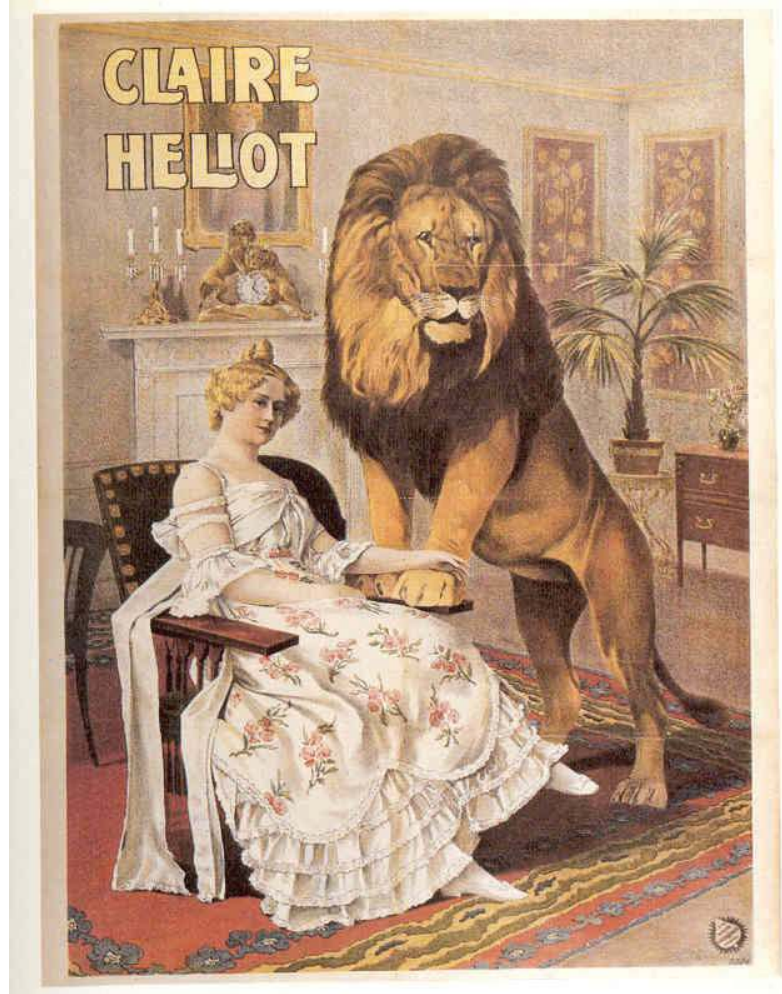

Adolph Friedländer, Claire Héliot, 1903, chromolithographie, 95 x $71 \mathrm{~cm}$, collection particulière (C) droits réservés

Assise sur son fauteuil, à côté d'un de ses lions, Claire Heliot apparaît élégante dans son intérieur fleuri et coquet. Elle montre une image d'elle qui dénote fortement avec ce que l'on pourrait attendre du cadre de vie d'une saltimbanque. Posté majestueusement à côté d'elle, le lion domestiqué se trouve quant à lui totalement soumis à la dompteuse qui a posé sa main sur sa grosse patte en signe de contrôle. Cette objetisation de l'animal, et le rapport de domination qui lui est associé, semblent se réitérer à l'arrière-plan de l'image, 
dans le décor au-dessus de la cheminée où le portrait encadré d'Heliot surplombe fièrement la pendule dorée figurant le combat entre deux fauves.

Mais d'autres représentations plus subversives se détachent de cette féminité respectable. Elles exacerbent les soupçons d'une possible déviance de la dompteuse dont les penchants lesbiens, sadomasochistes et même zoophiles seraient exaltés ${ }^{22}$. Une affiche du Cirque d'hiver montre, par exemple, la comtesse $\mathrm{X}$ entourée de ses fauves dans une attitude de dominatrice le téton pointé vers le haut, avec au centre de la poitrine une petite fleur jaune.

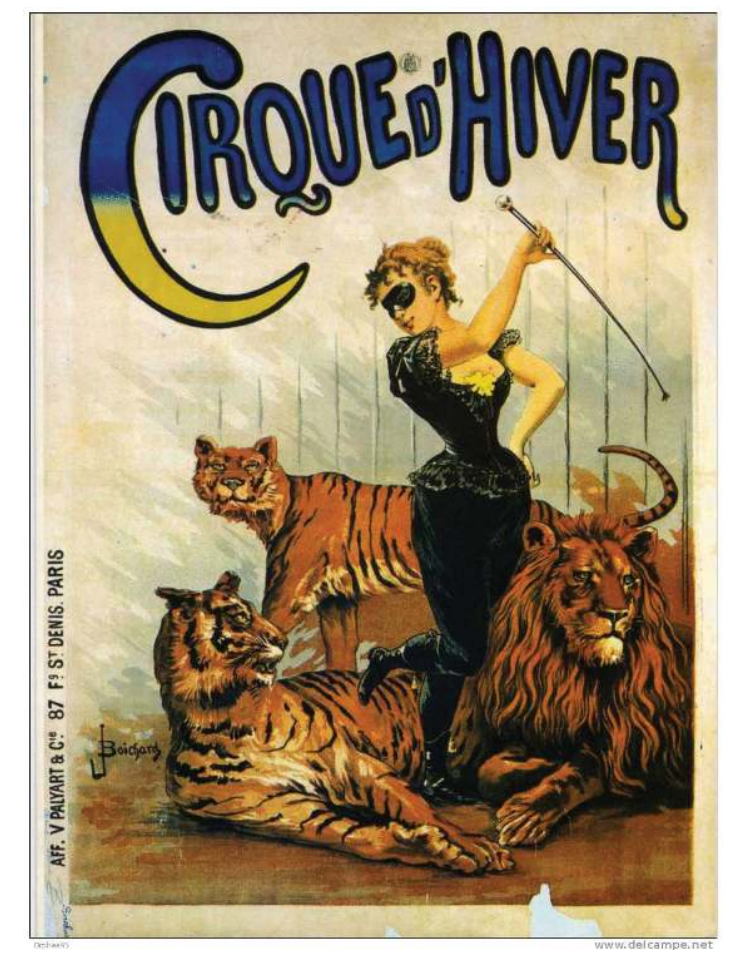

La comtesse de X (Mlle de Vésins) et ses fauves en 1900 au cirque d'hiver, affiche, collection particulière $(C)$ droits réservés.

Vêtue d'un corset noir, de bottes hautes, d'une cravache et d'un masque accessoires clairement connotés comme sadomasochistes -, cette dompteuse semble illustrer les textes décadents sur la sexualité de Richard Krafft-Ebing, Henry Havelock Ellis et Sigmund Freud qui vont contribuer à fabriquer une 
image dépravée d'un féminin associé à la passivité et à la soumission ${ }^{23}$. Si cette hypersexualité atteint parfois l'obscénité, comme dans une estampe d'Henri de Toulouse Lautrec - dont on connaît les liens étroits avec la Goulue - qui montre une dompteuse titillant à la cravache un fauve réduit à un phallus en érection ${ }^{24}$, elle ne doit pas non plus être considérée comme propre aux hommes. Ainsi, en 1908, la romancière Jane de La Vaudère brosse dans un roman le portrait d'une dompteuse prénommée Sapho et propose déjà, par le choix de ce nom, d'éveiller le trouble sur la sexualité de son héroïne. Dès les premières pages du récit, l'écrivaine décrit ainsi la relation passionnelle et équivoque que Sapho entretient avec sa panthère Mirah :

Durant un moment, Sapho l'étreint [la panthère noire Mirah], pose sa tête contre la sienne, et, mettant sa bouche sur le mufle crispé, semble s'oublier en un baiser profond. La panthère clôt ses paupières sombres, renverse le front, félinement ronronne, et se frôle à cette chair de femme tiède et parfumée. Ce sont vraiment deux bêtes d'amour, aussi câlines l'une que l'autre, griffes rentrées dans le gant de velours, regard perdu dans l'infini du rêve.

Puis, un coup de cravache atteint le fauve, le redresse, hurlant et terrible, gueule tendue, tous crocs dehors, dans le regret de la caresse ininterrompue, la rancune du plaisir mensonger. La femme rit, méprisante, et la bête humiliée se ramasse prête à fondre sur sa proie, à l'enserrer dans une étreinte sans pardon... Les deux corps vont rouler, s'embrasser, se mordre au milieu des râles et des rugissements. Quelques cris de femme se font entendre... un second coup de fouet, un regard fascinateur, un ordre bref et Mirah, soumise, vient ramper aux pieds de sa maîtresse, l'implorer pour un nouveau jeu très doux ${ }^{25}$.

\section{La dompteuse, une figure paradigmatique de l'émancipation} féminine

L'hypothèse que je souhaiterais formuler dans cet article est que l'ambivalence des genres attachée à la figure de la dompteuse est justement propice à une redéfinition d'une norme féminine. En instrumentalisant cette figure intermédiaire, certaines femmes vont en effet revendiquer une autre place, un autre statut, un autre rôle dans des sphères intellectuelles bien spécifiques. La dompteuse pourrait, à partir de là, devenir métaphore d'une revanche du féminin, une revanche qui trouve à s'exprimer, comme nous aimerions le suggérer, de deux manières stratégiques : l'excentricité et l'affirmation d'une hégémonie féminine. 


\section{Elles font leur cirque ! : la stratégie de l'excentricité}

Dans le Grand dictionnaire Larousse du XIx ${ }^{e}$ siècle, « excentricité » signifie autant bizarre, singulier, que le fait d'être hors du centre et à part ${ }^{26}$. Adopter une attitude excentrique est donc déjà adopter une attitude de rupture visà-vis d'une norme féminine préconçue. L'historienne Mary Louise Roberts précise, à ce sujet, que la célébrité des femmes est plus provocatrice à la fin du siècle que ne l'est celle des hommes, et qu'elle peut à ce titre être taxée d'excentricitée ${ }^{27}$. C'est ce type d'attitude singulière que nous retrouvons dans plusieurs photographies montrant la journaliste Marguerite Durand, l'artiste Rosa Bonheur, et la comédienne Cécile Sorel, posant à côté de fauves devenus leurs animaux de compagnie.

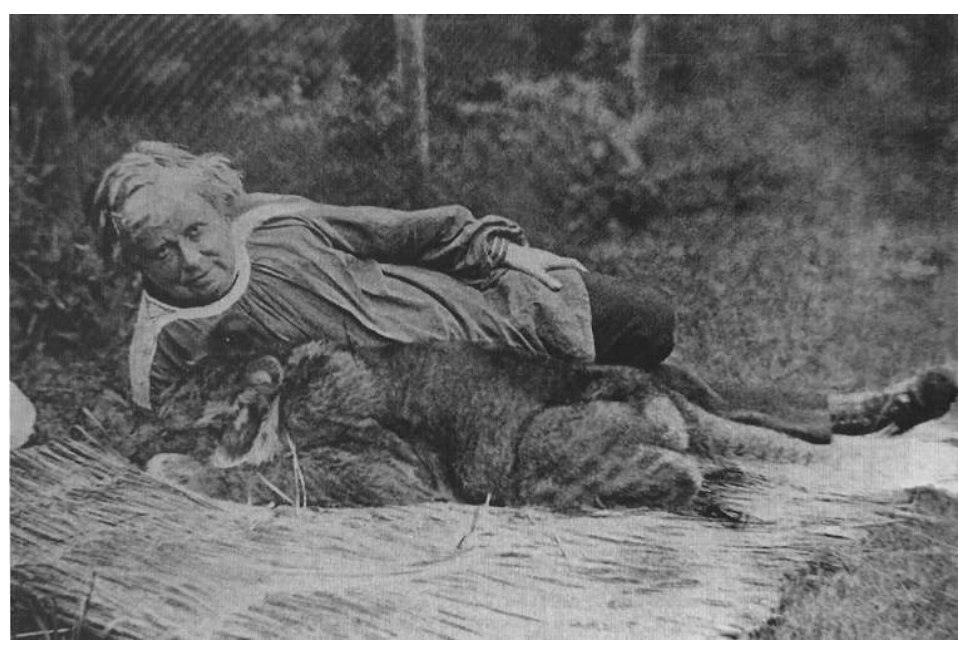

Photographie de Rosa Bonheur avec sa lionne Fatma, reproduite dans : Anna Klumpke, Rosa Bonheur, sa vie, son cuvre, Paris, Flammarion, 1908, p. 281.

Dans l'un de ces clichés, Rosa Bonheur privilégie plutôt une pose complice en étant allongée à côté de sa lionne Fatma et démontre par là qu'elle ne se contente pas uniquement de peindre des animaux mais qu'elle vit aussi en toute intimité avec eux. Cette promiscuité assumée avec l'animal sauvage pourrait rejoindre la thèse de Claire Nettleton qui compare l'artiste d'avantgarde au XIX ${ }^{\mathrm{e}}$ siècle à un animal en cage. Ce stéréotype s'associe non seulement à la lutte qu'il doit mener pour créer dans un contexte coercitif, marqué par les carcans institutionnels notamment, mais également à la valorisation dans sa pratique du retour à un œil primitif ${ }^{28}$. En faisant l'acquisition de lions, Rosa Bonheur pourrait ainsi reprendre à son compte cette grande liberté que ni un chat ni un chien, des animaux plutôt circonscrits au périmètre domestique, 
n'incarne. Comme la peintre Rosa Bonheur, l'actrice Sarah Bernhardt fait en 1895 l'acquisition d'un puma et d'un guépard ${ }^{29}$. L'excentricité de cet achat participe à un certain mode de vie éloigné des normes et des conventions, et peut permettre de rendre le féminisme plus attractif aux yeux des critiques. Posséder un animal est du même acabit - pour Bernhardt et Bonheur que vivre son homosexualité, porter le pantalon ou faire croire, dans le cas de la comédienne, qu'elle dort chaque soir dans un cercueil. Une façon de « faire leur cirque ! », pour reprendre l'expression convenue, c'est-à-dire se donner en spectacle, se mettre en scène, afin de provoquer le dérèglement des principes établis.

\section{Les reines de la piste : la stratégie de l'hégémonie}

Dans sa correspondance, l'écrivaine et peintre belge Emma Lambotte explique qu'elle aime se dépeindre « volontiers sous les traits d'une “dompteuse" $[\ldots]$ indépendante jusqu'à la révolte, personnelle jusqu’à la férocité $^{30} \gg$. Il semble ici que la dompteuse intéresse Lambotte par sa capacité de lutte qui peut rejoindre le combat qu'elle mène pour parvenir à exister en tant que femme dans les sphères artistique et littéraire. Le souci de réussite dans ces milieux est aussi ce qui caractérise le dessin de Gustave Verbeck intitulé « Le Tigre critique d'art » qui montre, sous un angle humoristique, la victoire d'une femme artiste à l'issue d'un affrontement mené avec un animal sauvage. 


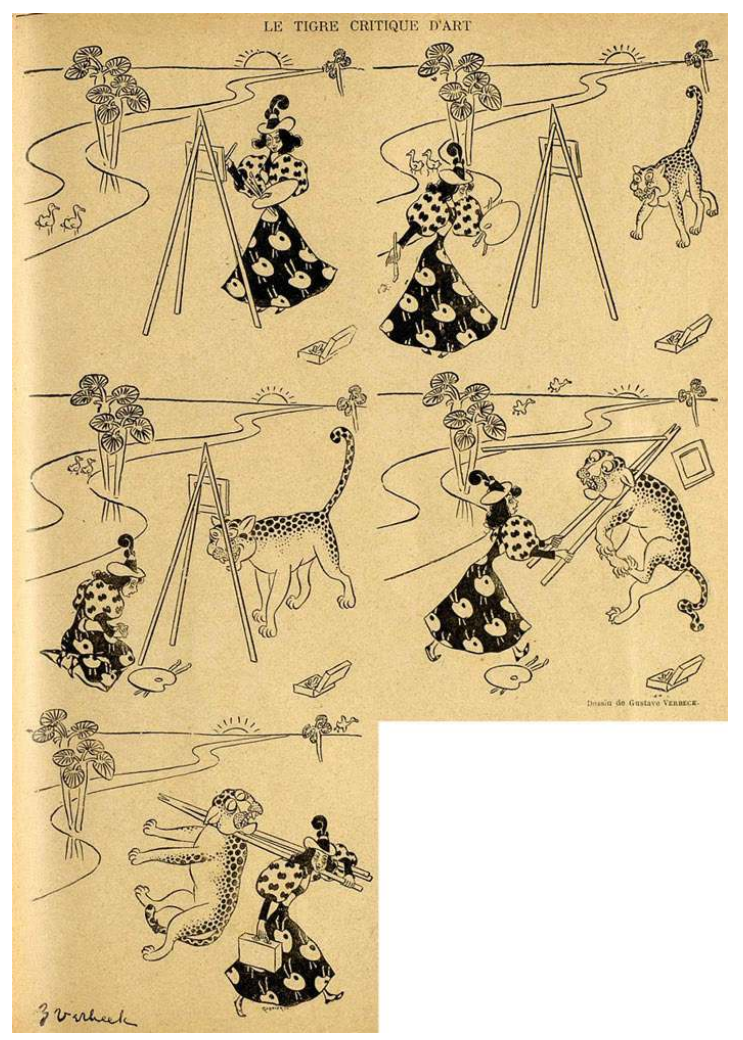

Alfred Grévin, Petit Journal pour rire, n² 236, 1880, une.

Ce dessin illustre en cinq scénettes la confrontation d'une femme tranquillement occupée à peindre deux canards dans un paysage, avec un tigre qui, les yeux écarquillés et les crocs sortis, est happé par la représentation alléchante des deux animaux peints par l'artiste. Si la peintre est, dans un premier temps, apeurée par le fauve qui vient la troubler dans son travail, elle parvient toutefois, à l'issue d'un combat rudement mené, à dominer l'animal telle une braconnière, en lui coinçant la tête entre les tréteaux de son chevalet.

La figure du tigre qui, dans le dessin de Verbeck reste animale, est plus clairement humanisée dans la caricature d'Alfred Grévin publiée dans Le Petit Journal pour rire en 1880 au moment du Mardi gras. 


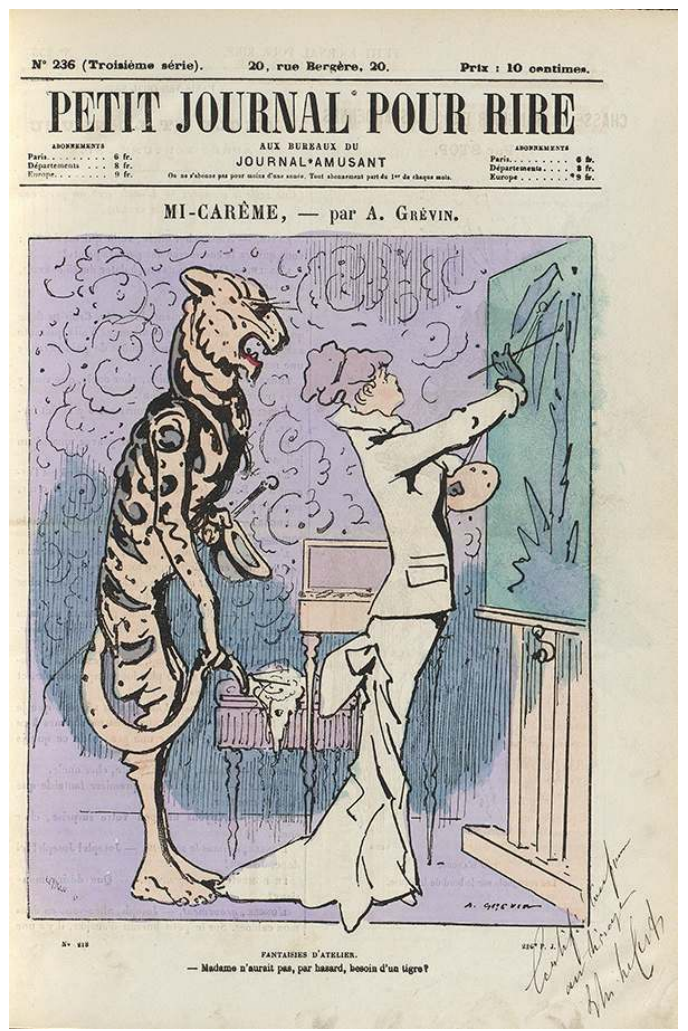

Gustave Verbeck, « Le Tigre critique d'art », Le Rire, n 35, 6 juillet 1895, p. 3.

Le dessinateur utilise ici le mécanisme du travestissement pour révéler la difficulté qu'ont les hommes à trouver leur place face à l'intrusion des femmes dans des domaines qui leur étaient auparavant réservés comme la peinture. $\mathrm{Si}$ le modèle masculin est déguisé en tigre affaibli, loin de l'image traditionnelle du fauve dominateur, il s'oppose plus nettement à la femme artiste dont le corps étiré et les membres raidis forgent l'image d'une dompteuse d'animaux, le pinceau pointé tel un bâton de dressage, dirigeant la scène d'une main de maître. Ces dessins satiriques donnent ainsi de l'émancipation féminine un nouveau visage subversif, le visage du rire, et anticipent, en quelque sorte, l'incantation de Judith Butler pour qui, « face à des catégories sérieuses, le rire est indispensable pour le féminisme ${ }^{31} \gg$.

Comme allant à l'encontre de cette prescription butlérienne, la comédienne Cécile Sorel et la patronne du journal féministe La Fronde, Marguerite Durand se montrent plus austères et sérieuses en trônant en majesté à côté 
de leurs bêtes. Debout et altières, dominant fièrement l'animal amadoué et couché à leurs pieds qui, quant à lui, pourrait apparaître comme un substitut masculin et illustrer une masculinité en crise $^{32}$, elles incarnent symboliquement le rayonnement majestueux des femmes dans les sphères intellectuelles et créatives. Cette idée semble d'ailleurs se confirmer avec les déclarations de Cécile Sorel qui explique qu'elle a « toujours été charmée par ce qui est beau, ce qui est grand, ce qui est fort ${ }^{33} \gg$, ou encore Marguerite Durand qui choisit non seulement d'appeler sa lionne « tigre »- affirmant par le changement sémantique une inversion des genres toute symbolique - mais également de dissimuler dans sa photographie à la une de Femina, le $1^{\text {er }}$ avril 1910, un homme uniquement visible en transparence derrière une vitre, comme pour faire allusion à la disparition progressive de l'autorité masculine.

Dans son ouvrage Wild and Dangerous Performances. Animals, Emotions, Circus paru en 2012, la chercheure australienne en performance studies Peta Tait pointe dans l'un de ses chapitres une articulation intéressante entre l'identité animale et la conquête de nouveaux droits féministes et raciaux ${ }^{34}$. Les travaux respectifs de Peter Singer et de Carol J. Adams au sujet de la cause animale $^{35}$ lui permettent de confirmer l'existence de points de convergence entre les mouvements de libération animale et les mouvements de libération des femmes dans les années 1960-1970 : l'égalité entre les animaux, la déhiérarchisation des espèces, la promotion de l'amour, du plaisir, au détriment de l'humiliation et de la souffrance... L'animal - et l'animal sauvage en particulier - aurait ainsi nourri les dynamiques intersectionnelles de race et de genre ; Tait mettant là en évidence, à l'appui de plusieurs arguments et exemples, que l'Autre et l'émotionnel forment des notions qui peuvent aussi bien être féminisées que racialisées. Par exemple, en important en Europe et aux États-Unis des animaux d'Asie, tels que le tigre du Bengale, ou venant de la jungle africaine, les professionnels du cirque ont surtout privilégié des espèces qui n'appartenaient pas à leur territoire et à leur culture ; une façon sans doute de mieux affermir la suprématie et l'impérialisme de leur propre continent. Cette manière très « andro-européano-humano-centrée » de régner se retrouve aussi dans une certaine façon de penser les sciences et les savoirs biologiques, si l'on songe aux processus plutôt rigides de classification des espèces et de taxonomies ou à l'héritage darwinien dans la construction linéaire et positiviste d'une histoire des espèces vivantes. À partir de ces éléments qui caractérisent un univers où l'homme occidental paraît régner en maître, Peta Tait suggère que les dompteuses sont finalement parvenues 
à redéfinir les pratiques de domptage sous un angle autre, plus émotionnel que rationnel, en se focalisant sur un lien empathique et nourricier à l'animal, comme en développant la prise de risques physiques et l'excitation face au danger.

Avec la figure circassienne de la dompteuse, qui permet à plusieurs femmes soucieuses d'émancipation de défier les hommes sur leurs propres territoires, le féminisme de la première vague semble anticiper de quelques années ces points de contact extrêmement stimulants de la seconde moitié $\mathrm{du} \mathrm{Xx}^{\mathrm{e}}$ siècle. Il est par ailleurs indéniable que le caractère spectaculaire, sensationnel et clinquant des attractions du cirque s'accommode bien de ce jeu de subversion des normes, que l'on songe aux bêtes de foire ou au clown travesti. Le détour par le cirque paraît effectivement faciliter ce travail de dévoilement, de déstabilisation, d'exhibition, de négociation et de performativité des identités, comme le montre bien l'anthropologue Christian Lallier. Ce dernier propose une définition tout à fait pertinente à la lumière des profils de femmes entraperçus dans cet article, quand il fait reposer l'activité des gens du cirque sur le contrôle : non seulement le « contrôle de soi », car il s'agit de mettre en spectacle la maîtrise de son corps, de sa technique et de ses risques, mais également le contrôle comme un « contre-rôle » de soi-même, au sens d'adopter une position réflexive et oppositionnelle au rôle social tel qu' il est traditionnellement délimité et attendu ${ }^{36}$.

\section{Notes}

1. Cet article est le fruit d'une communication donnée au colloque international «Genre et création dans l'histoire des arts vivants », organisé du 12 au 14 décembre 2013 par Elizabeth Claire, Catherine Deutsch et Raphaëlle Doyon. Il constitue une version approfondie et enrichie d'un sous-chapitre de ma thèse de doctorat soutenue en 2012 et disponible depuis sous la forme d'un essai : Charlotte Foucher Zarmanian, Créatrices en 1900. Femmes artistes en France dans les milieux symbolistes, Paris, Mare \& Martin, 2015.

2. Voir notamment Peta Tait, Circus Bodies. Cultural Identity in Aerial Performance, Londres, Routledge, 2005. Sur l'écuyère : Paul Adrian, Le cirque commence à cheval, Paris, Adrian, 1979, p. 36 : « Sœur du voltigeur et de la voltigeuse, elle est la ballerine à cheval. Elle demeure l'une des plus gracieuses incarnations du travail équestre debout. [...] Avec elle toute la force devient charme : jeune, agréable à regarder, souriante, elle danse sur le dos nu du cheval, ou ce qui est rare de nos jours, sur une grande selle plate ». Ibid., p. 54 : « La dame du cirque, celle qui plus que tout autre femme de la piste, apportait, dans un spectacle qui parlait plus aux yeux qu'au cœur, une note presque sentimentale. Alors quétaient simplement applaudies l'acrobate, la funambule, voire la dompteuse, 
l'écuyère était aimée. Conteurs et romanciers trouvèrent en elle une belle source d'inspiration romantique. "Voir Octave Mirbeau, «L'Écuyère », Euvres romanesques, Paris, Buchet/Chestel, Pierre Zech éditeur, 2000 [publié en 1882 sous le pseudonyme d'Alain Bauquenne], p. 789-967 ; Paul Bourget, L'Écuyère, Paris, Plon Nourrit, 1921. Sur cette figure de l'écuyère, voir l'étude de Tristan Rémy, Écuyères romantiques. La belle Madame Lejars et ses scurs Pauline et Armantine, Paris, Cirques, 1960.

3. Voir à ce sujet Magali Sizorn, "Transformation des arts du cirque et identités de genre ", STAPS n ${ }^{\circ}$ 61,2003/2, p. 11-24: « Le cirque traditionnel continue à diffuser des schémas de relation où hommes et femmes restent à une place sexuellement pré-définie. Leur relation est "hyper-ritualisée" : ils incarnent d'une façon manichéenne le masculin (lui) et le féminin (elle). Le couple "sur-ritualise" les relations hommes-femmes : lui est fort, et elle est femme-enfant, femme-soumise ou femme-séductrice. »

4. Voir Henry Thétard, "Chapitre VI : Dompteuses et reines de lions ", Les Dompteurs ou La Ménagerie des origines à nos jours, Paris, Gallimard, 1928, p. 141. Voir également du même auteur «Les reines des fauves ", Revue des deux mondes, 15 mars 1954, p. 325-340.

5. Voir Cesare Lombroso, Guglielmo Ferrero, La Donna delinquente, la prostituta e la donna normale, Turin, Roux, 1893. Louvrage a paru pour la première fois en France aux éditions Félix Alcan en 1896, mais nous nous appuyons ici sur son édition la plus récente, à savoir : La Femme criminelle et la prostituée, Grenoble, Jérôme Millon, 1991, p. 83-108.

6. Ibid., p. 29.

7. Cité dans : Bram Dijkstra, Les Idoles de la perversité. Figures de la femme fatale dans la culture fin de siècle, Paris, Seuil, 1992 [Oxford, Oxford University Press, 1986], traduction française par Josée Kamoun, p. 167. Voir L'argument naturaliste est également avancé dans la littérature de Charles Baudelaire qui écrit dans Mon cœur mis à nu que « la femme a faim et elle veut manger. La femme est naturelle c'est-à-dire abominable. [...] Elle est simpliste comme les animaux ", dans Charles Baudelaire, Euvres complètes. Préface, présentation et notes de Marcel A. Ruff, Paris, Seuil, 1968 [1863], p. 406.

8. Henri Cazalis, Henri Régnault, sa vie et son œuvre, Paris, Alphonse Lemerre, 1872, p. 74.

9. Voir Miss Nouma Hawa, Mémoires d'une dompteuse écrits par elle-même. L'art de dompter les animaux féroces et de charmer les serpents, [s. 1.], [s. n.], 1880.

10. Voir Gladys la dompteuse, 1920, comédie dramatique en quatre parties, Paramount Pictures, Paris, $\mathrm{BnF}$ arts du spectacle, $8^{\circ} \mathrm{Rk} 4784$.

11. Paul Rousselot, Histoire de l'éducation des femmes en France, vol. 2, Paris, Didier, 1883, p. 428. Voir également l'anthologie de Jean Houssaye (dir.), Femmes pédagogues, t. I, De l'Antiquité au XIXe siècle, Paris, éditions Fabert, 2008. Cornaline la dompteuse parle, par exemple, de « ses élèves dans la cage " pour désigner ses fauves. Voir Fortuné Du Boisgobey, Cornaline la dompteuse, Paris, Librairie Plon, 1887, p. 67.

12. Voir Jacob Smith, The Thrill Makers: Celebrity, Masculinity and Stunt Performance, Los Angeles, University of California Press, 2012, p. 96.

13. Voir Franck C. Bostock, Le Dressage des fauves, Paris, Hachette, 1904, traduction française depuis l'anglais [dans les pages qu'il consacre à la dompteuse Louise Morelli] : «Les léopards, les jaguars et les panthères se distinguent par leurs habitudes astucieuses et furtives, par leur perfidie et leur ruse. Ils sont fort difficiles à maîtriser et à dresser, et 
l'on ne peut jamais se fier à eux. Ces grands chats rampants ont la nature des démons les plus méchants, ils n'ont rien de la noblesse du lion, rien de la réserve du tigre. »

14. Voir également le cas contemporain d'une jeune anglaise parvenant à maîtriser un lion qui s'était auparavant attaqué avec violence à un dompteur. Voir [Anonyme], «Les lions ne sont jamais aussi domptés qu'on croit ", La Vie heureuse n 12, décembre 1903, p. 223-224 : «Un jour, un dompteur fut saisi par un lion et promené comme la souris l'est par le chat. Heureusement la dompteuse qui l'accompagnait eut la présence d'esprit de saisir le lion du geste caressant que cette image représente. À ce geste le lion avait l'habitude de se coucher. Il lâcha l'homme et exécuta un mouvement accoutumé. »

15. Émile Littré, Dictionnaire de la langue française, t. II, Paris, Librairie Hachette et Cie, 1885, p. 1213.

16. Ernest Grenet-Dancourt, Georges Bertal, Norah la dompteuse. Vaudeville en trois actes, représenté pour la première fois à Paris sur le Théâtre des Nouveautés le 31 octobre 1891, Paris, Didier \& Méricant, [1891].

17. Ibid., p. 63.

18. Ibid., p. 88.

19. Voir «Une dompteuse dévorée par une lionne (Mort de Mlle Rosita Gondolfo, à Grenoble) ", Le Petit Journal. Supplément illustré, samedi 25 avril 1891 (une) ; " Drame sanglant dans une ménagerie (La Goulue et son mari aux prises avec un puma) », Le Petit Journal. Supplément illustré, 24 janvier 1904 (une).

20. Voir Nichola Haxell, "Ces Dames du Cirque": A Taxonomy of Male Desire in Nineteenth-Century French Literature and Art ", MLN. French Issue, septembre 2000, vol. CXV, p. 783-800.

21. Fortuné Du Boisgobey, Cornaline la dompteuse, op. cit., p. 32.

22. La pratique zoophile est sous-entendue dans la courte nouvelle de Catulle Mendès, «Les deux lionnes », Bêtes roses, Paris, Librairie Nilsson - Per Lamm, 1899, p. 147-151, où le palefrenier Ragot entre dans la cage de la lionne Rosette, perturbée par l'orage et en période de rut, pour la satisfaire.

23. Voir Alison Moore, "Rethinking Gendered Perversion and Degeneration in Visions of Sadism and Masochism, 1886-1930 ", Journal of the History of Sexuality, vol. XVIII, $\mathrm{n}^{\circ} 1$, janvier 2009, p. 138-157.

24. Voir Henri de Toulouse-Lautrec, Dompteuse, [s.d.], dessin, localisation actuelle inconnue.

25. Jane de La Vaudère, Sapho, Dompteuse. Roman, Paris, Albert Méricant, 1908, p. 6-7.

26. Pierre Larousse (dir.), Grand Dictionnaire universel du XIX siècle, t. 7 (E), Paris, Administration du grand Dictionnaire universel, 1866-1877, p. 148-149.

27. Voir Mary Louise Roberts, "Rethinking Female Celebrity. The Eccentric Star of Nineteenth-Century France ", Constructing Charisma. Celebrity, Fame, and Power in Nineteenth-Century France, New York, Oxford, Berghahn Books, 2010, p. 103.

28. Voir Claire Nettleton, "The Caged Animal. The Avant-Garde Artist in Manette Salomon ", Antennae. The Journal of Nature in Visual Culture n ${ }^{\circ}$ XXIV, p. 75-90. Son argumentaire prend notamment appui sur le roman Manette Salomon des frères Goncourt dont les dernières pages sont consacrées à l'artiste Anatole Bazoche qui devient gardien de zoo au Jardin des Plantes. Avec son collègue Coriolis, Bazoche a l'habitude d'aller au Jardin des Plantes pour peindre les animaux de la ménagerie. 
29. Sarah Bernhardt, qui possède un puma dès 1895 , fréquentait lors de son séjour à Londres, " pendant ses loisirs, [...] les zoos, allant jusqu'à Liverpool et ramenant des fauves : un guépard, un chien-loup, qui la nuit alarmèrent le paisible quartier de Chester Square qu'elle habitait ", dans Ernest Pronier, Sarah Bernhardt. Une vie au théâtre, Genève, éditions Alex. Julien, [1946], p. 67.

30. Laurent Tailhade, "Préface ", Les Roseaux de Midas, Emma Lambotte (éd.), Paris, Vanier, 1910, p. XV, cité dans : Laurence Brogniez, "Poétesses belges du XIX ${ }^{\mathrm{e}}$ siècle : quelques profils perdus ", Sextant. Revue du groupe interdisciplinaire d'études sur les femmes $\mathrm{n}^{\circ} 17-18,2002$, p. 147.

31. Judith Butler, Trouble dans le genre (Gender Trouble). Le féminisme et la subversion de l'identité, Paris, La Découverte/Poche, 2006, traduction française par Cynthia Kraus [New York - Londres, Routledge, 1990], p. 52.

32. Voir Christopher E. Forth, The Dreyfus Affair and the Crisis of the French Manhood, Baltimore, Johns Hopkins University Press, 2004 ; Annelise Maugue, L'Identité masculine en crise au tournant du siècle, 1871-1914, Paris, Rivages/Histoire, 1987 ; André Rauch, Crise de l'identité masculine 1789-1914, Paris, Hachette Littératures, 2000.

33. Voir Cécile Sorel, « $\mathrm{M}^{\text {1le }}$ Cécile Sorel nous parle de ses lions ", Femina n ${ }^{\circ} 227,1^{\text {er }}$ juillet 1910, p. 350.

34. Peta Tait, "Leopard around the Neck ", Wild and Dangerous Performances. Animals, Emotions, Circus, Londres, Palgrave Macmillan, 2012, p. 141-146.

35. Voir Peter Singer, Animal Liberation, Londres, Pimlico, 1995 [1975] ; Carol J. Adams, Neither Man Nor Best: Feminism and the Defense of Animals, New York, Continuum, 1994.

36. Voir Christian Lallier, "Le cirque, la performance du contre-rôle de soi ", Francine Fourmaux (dir.), Les Lieux du cirque. Du local à l'international, trajectoires et inscription spatiale des circassiens, Paris, Le Manuscrit, 2008, p. 155. 